\title{
High-Fidelity Passive Force-Reflecting Virtual Environments
}

\author{
Mohsen Mahvash and Vincent Hayward, Senior Member, IEEE
}

\begin{abstract}
Passivity theory is applied to the creation of synthetic, complex multidimensional haptic environments. It can be shown that under appropriate conditions, sufficiently high rendering rates can guarantee the passivity of a simulation produced by a haptic device coupled to a discrete-time realization of a nominally passive environment. The creation of a passive, globally defined, virtual environment is either analytically complex or computationally costly. A method is described whereby a passive environment is created from transitions between locally defined force models that encode static conservative force fields. This is applied to the haptic rendering of tool contact with deformable bodies, in which sparse force-deflection responses are used to define local models. Passivity, continuity, and fidelity are provided by response-function interpolation rather than by interpolation of forces, as in previous methods. The paper also includes an illustrative example.
\end{abstract}

Index Terms-Deformable bodies, force-reflective virtual environments, haptic simulation, passivity.

\section{INTRODUCTION}

$\mathbf{F}$ ORCE reflection has many applications, such as in surgical training simulators, computer-aided design systems, and virtual environments. A simulated environment with force reflection is characterized by a tightly closed loop. From a stability viewpoint, for a virtual interaction without force, bounded-input bounded-output (BIBO) stability of the virtual environment is sufficient to prevent the explosion or unwanted oscillations of a simulated graphical scene. However, even with BIBO stability, self-sustaining oscillations can develop while interacting with a force-reflecting virtual environment.

Passivity is a physical property of most environments of interest. A correspondingly passive force-reflecting virtual environment not only more closely simulates the energy response of a real environment, but also guarantees the stability of the interactions during which the user does not intend to destabilize the interaction. However, the discrete-time simulation of a nominally passive continuous-time environment generally becomes active. We show that with a sufficiently high update rate, the discrete-time simulation of a passive continuous-time environment

Manuscript received January 14, 2004. This paper was recommended for publication by Associate Editor C. Melchiorri and Editor H. Arai upon evaluation of the reviewers' comments. This work was supported in part by the project "Reality-based Modeling and Simulation of Physical Systems in Virtual Environments" of the Institute for Robotics and Intelligent Systems (IRIS) (Canada's Network of Centers of Excellence). The work of V. Hayward was supported by the Natural Sciences and Engineering Council of Canada (NSERC) in the form of a discovery grant.

M. Mahvash was with the Center For Intelligent Machines, McGill University, Montréal, QC H3A 2A7, Canada. He is now with RealContact Inc., Montréal, QC H2X 2B1, Canada.

V. Hayward is with the Center For Intelligent Machines, McGill University, Montréal, QC H3A 2A7, Canada (e-mail: hayward@ cim.mcgill.ca).

Digital Object Identifier 10.1109/TRO.2004.833819

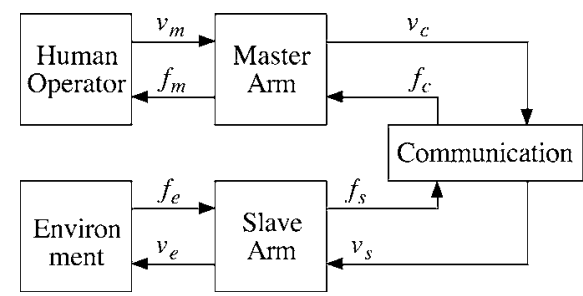

Fig. 1. Two-port block representation of teleoperation systems.

results in a passive force-reflecting virtual environment synthesized at the end-effector of a haptic device.

The global determination of the forces produced during virtual interaction requires costly computations and/or the use of high-order analytical solutions. For example, the haptic rendering of a deformable body using a finite-element method (FEM) or boundary-element method (BEM) requires the use of "numerical compression," because of the rapid growth of the computational requirements with problem size [4]. To overcome these problems, low-order local models and multirate simulations are often proposed [4], [6], [8], [14], [18]. However, the force response resulting from switching between local models does not generally guarantee passivity, continuity, and prescribed accuracy.

We introduce a force-update method in which a passive simulated environment is obtained from locally defined passiveforce models activated in sequence. The proposed force-update strategy essentially prevents any increase of the potential energy of the virtual environment at the moment of activation.

The method is applied to high-fidelity haptic rendering of tool contact with a deformable body, in which actual force responses are used to create locally defined models by reproducing them exactly. The simulated deformable body can be nonlinear elastic, anisotropic, and inhomogeneous, as described in greater detail in [16]. An implementation is also described.

\section{RELATED WORK}

Anderson and Spong pioneered the use of passivity theory in teleoperation systems. They introduced the basic notions needed to analyze force-feedback systems in terms of passivity [2]. A complete system was divided into four passive subsystems, including the human operator, the master arm, the slave arm, the environment, plus a nonpassive communication block, see Fig. 1. By active control, the communication block was changed to a passive system. The passivity of the teleoperation subsystems led to the stability of the system. Niemeyer and Slotine adopted a similar framework, and successfully used the notion of wave variables to provide stability, as well as explicit design tradeoffs regarding fidelity [22]. This framework was also used by Yokokohji et al. to deal with fluctuating time delays [26]. 
This paper adopts these notions developed for teleoperation and uses them for haptics, considering that the remote environment, the slave robot, and the communication link are replaced by a force-reflecting computational environment. Thus, the five basic subsystems of teleoperation are replaced by three in haptics, as seen in the next section.

Colgate and Shenkel derived a necessary and sufficient condition for the passivity of a haptic interaction system comprising a linear dissipative device, a discrete-time virtual environment of a certain class, and a linear user model [7]. It was shown that if a device is modeled by a damper $b$ and a mass $m$ interacting with a virtual wall constructed from a spring $k$ and a damper $B$, then the system is passive when $b>(k T / 2)+B$, where $T$ is a sampling period. This means that a higher sampling rate allows for a stiffer passive wall, while the virtual damping is always limited to device damping. Miller et al. later derived a sufficient condition for haptic interaction stability for a broad class of nonlinear and time-delayed virtual environments, including nonlinear masses, springs, and dampers, when the device and user models were linear [21]. This paper extends this result to nonlinear multidimensional virtual environments and nonlinear devices. However, we must assume the existence of an upper bound for interaction acceleration, and of some dissipation in the device, conditions that are always ensured in practice.

A virtual coupling block constructed from springs and dampers is often interposed between the virtual environment and the device to provide interaction stability [1], [5], [21]. The virtual coupling limits the stiffness of the rendered virtual environment, and modifies the damping parameters of the nominal environment. Recently, Hannaford and Ryu employed a time-domain passivity strategy involving a passivity observer coupled to a time-varying passivity controller designed to inject damping into the system [11]. In all these approaches, fidelity may be compromised to gain passivity, since a subsystem is added that was not specified in the original simulation. Another approach was proposed by Stramigioli et al. [25], but it is not clear how it can be applied to nonlinear multidimensional virtual environments.

The excellent survey by Salisbury et al. describes many important issues in haptic rendering, including the need for fidelity in some applications [24]. The authors suggest that calculating forces from a potential field can provide passive contact surfaces for three-dimensional virtual bodies, but the design of these fields, in general, was left as an open question. The method introduced in this paper is inspired by the "god object" method, which implicitly uses locally defined passive-force fields, and possibly passive switching, for haptic rendering for the case of rigid bodies [27]. Here we extend this idea to apply it to the simulation of deformable bodies that can be nonlinear elastic, anisotropic, and inhomogeneous.

\section{PASSIVITY IN HAPTIC INTERACTION SYSTEMS}

\section{A. Definitions}

Following [15] (positive power flowing in the system).

Definition 1: A system with flow $v$, effort $f$, and initial energy $E(0), v(t), f(t) \in \mathbb{R}^{n}$ is passive if

$$
\int_{0}^{t} f(\tau)^{\top} v(\tau) d \tau+E(0) \geq 0
$$

for all functions $v, f$, and for $t \geq 0$.
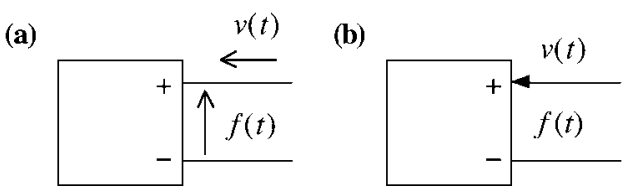

Fig. 2. A one-port block element. In (a), arrows indicate how the variables of flow and effort are labeled. The more concise labeling, as in (b), is used.

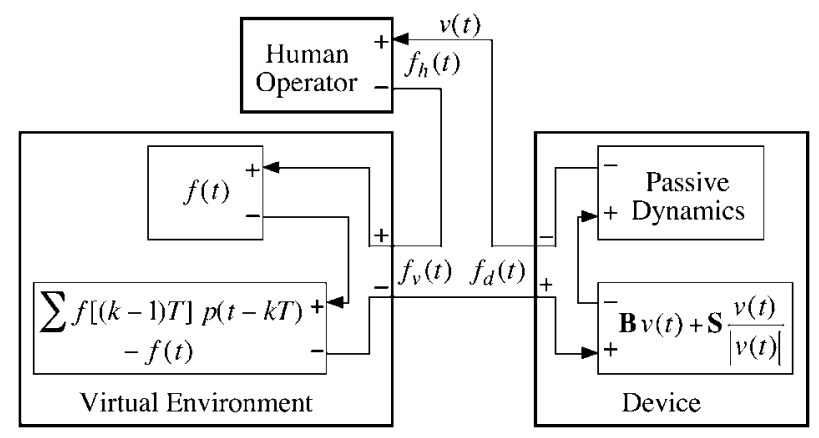

Fig. 3. One-port block representation of haptic interaction systems.

The one-port block representation (see Fig. 2) is convenient for the purpose of this paper, since it makes it possible to decompose a system in the manner of a circuit network. Here, a mechanical force $f(t)$ is the variable of effort measured between two terminals + and - , and a velocity $v(t)$ is the variable of flow through the block element. With this notation, two blocks mechanically connected to each other have the same velocity, and hence, are connected "in series." The variables of effort add up to zero in closed circuits, and hence, circuits represent force-balance equations. If needed, this representation can be converted to input-output system diagrams using Kirchkoff's Laws.

For a vector in $\mathbb{R}^{n},|\cdot|$ means here the Euclidian norm $\|\cdot\|_{2}$. If the haptic interaction can be described with just a force or just a torque, then $|f|$ has the ordinary meaning of magnitude. If the haptic interaction combines forces and torques, then $|f|$ no longer has a physical meaning. This is, as often discussed, because its value depends on arbitrary choices of units and coordinate frames. However, Theorem 1 in the next section is an existence theorem that only requires the existence of $|f|$ (and of $|\Delta x|)$, but not unicity. It is possible to give physical meanings to - $\mid$ by using weights and well-chosen coordinates, but this is not needed here. Note, however, that expressions like $f^{\top} v$, with $v$ a vector of linear and angular velocities, always represent power.

A haptic interaction system generally comprises an actuated mechanism in mechanical contact with a person. The mechanism is connected to a computer running a simulation. Using one-port blocks, any such system can be represented by the circuit shown in Fig. 3. It expresses the fact that the velocity $v$ of the contact interface at the handle is common to the operator, to the device. In addition, all forces balance at the contact interface between the device and the operator. Call $f_{h}$ the force exerted by the human operator, $f_{v}$ the force produced by the simulation via actuators driven by digital-to-analog converters, and $f_{d}$ the forces caused by the dynamics of the device, then $f_{h}+f_{v}+f_{d}=0$.

The complete system is divided into three subsystems, which are each discussed in turn. 
Human operator. No assumption is made about this subsystem, because we seek to provide fidelity to the artificial part of the interaction. Fidelity means that the artificial environment should share certain properties with a real environment, the most important of which being passivity, but nothing needs to be said about the human operator. The stability of the system as a whole is neither implied nor required. To illustrate this, we observe that certain cases of interaction with a passive environment are unstable. A drum roll, for example, is a rebound-based sustained oscillation. At the contact between the stick and the drum head, the equilibrium is not stable, in the sense of Lyapunov. The drum and stick are passive, nevertheless, and each obey condition (1). Clearly, exactly the same environment can also yield a stable interaction.

Device. This block represents the device, excluding force transduction (e.g., Lorentz Law). This corresponds to the notion of "ground device" or unpowered device [13]. From physics, there must always be some dissipation. No matter how well constructed, a device has surfaces sliding past each other. If it is levitated [23], there must exist magnetic losses, and so on. A way to express this is to state the existence of dissipative forces. In other terms, we can always find positive-definite matrices $\mathbf{B}$ and $\mathbf{S}$, no matter how small, that bound viscous and dry friction. Ignoring other forms of dissipation, and using the division sign for element-wise division

$$
\exists \mathbf{B}, \mathbf{S} \geq \mathbf{0} \text { s.t. }\left|f_{\text {diss }}\right|>\left|\mathbf{B} v+\mathbf{S} \frac{v}{|v|}\right|, \quad v \neq \mathbf{0} .
$$

Again, $\mathbf{B}$ and $\mathbf{S}$ do not have to be unique nor be physically meaningful, that they exist is what matters. A device can, therefore, be represented by a dissipative subblock plus a passive subblock representing other dynamics (possibly with more dissipation).

Virtual Environment. It can also be viewed as a block element with velocity signal $v(t)$ related to the measurements made by the haptic device. This velocity is associated with a force $f_{v}(t)$ resulting from a discrete-time simulation, and with $f(t)$, the corresponding continuous-time signal that the simulation represents. That these two signals are not the same is cause for loss of passivity of a complete system, even when the combination of $v(t)$ and $f(t)$ represents a passive system, termed here the "nominal system." This spurious energy production was called "energy leak" [10], or "energy error" [9]. We can express the combined effects of zero-order hold conversion and computational delay by

$$
f_{v}(t)=\sum_{k} f[(k-1) T] p(t-k T)
$$

where $T$ is the sampling period and $p(t)$ is a pulse

$$
p(\tau)= \begin{cases}1, & 0 \leq \tau<T \\ 0, & \text { otherwise }\end{cases}
$$

$f_{v}(t)$ can also be written as $f(t)+\left[f_{v}(t)-f(t)\right]$ to represent the virtual environment as the series connection of two subblocks, as shown in Fig. 3. This way, the second subblock essentially models the effect of zero-order hold conversion and delay.

Next, we reorganize the system of Fig. 3, so that the dissipative subblock and the subblock which represents the effect

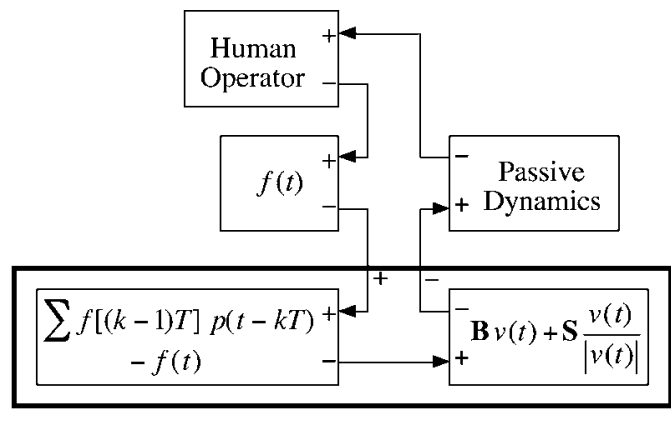

Fig. 4. Reorganization of the system of Fig. 3.

of delay and discretization are lumped into one subsystem, as shown in Fig. 4. Given that the device dynamics must always be passive, the combination of the virtual environment and the device will be passive if 1) the nominal system is passive, and if 2) the subsystem in outlined in grey in Fig. 4 is passive.

We now investigate the passivity of the subsystem outlined in Fig. 4. From now on, let $t_{k}=k T$.

\section{B. Existence of Passivity}

Theorem 1: Given the subsystem of Fig. 4 during $t_{k-1} \leq$ $t<t_{k} \forall k>0$, if

1) given $\sigma_{0}(\mathbf{B})$ the smallest singular value of $\mathbf{B}$, and $x(t)$ the position of the haptic device

$$
\left|f(t)-f\left(t_{k-1}\right)\right| \leq \frac{\sigma_{0}(\mathbf{B})}{2 T}\left|x(t)-x\left(t_{k-1}\right)\right|
$$

2) given $a(t)$ the interaction acceleration, and $\sigma_{0}(\mathbf{S})$ the smallest singular value of $\mathbf{S}$

$$
|a(t)| \leq \frac{\sigma_{0}(\mathbf{S})}{T \sigma_{0}(\mathbf{B})}
$$

3) $v(t)$ is $C^{0}$ continuous

then there exists $T>0$ such that the subsystem is passive.

Proof: For $t_{k-1} \leq \tau<t_{k}$, Condition 1) yields

$$
\left|f(\tau)-f\left(t_{k-1}\right)\right| \leq \frac{\sigma_{0}(\mathbf{B})}{2 T}\left|x(\tau)-x\left(t_{k-1}\right)\right| .
$$

Multiplying both sides of (7) by $|v(\tau)|$ gives

$$
\left|f(\tau)-f\left(t_{k-1}\right)\right||v(\tau)| \leq \frac{\sigma_{0}(\mathbf{B})}{2 T}\left|x(\tau)-x\left(t_{k-1}\right)\right||v(\tau)| .
$$

The inner product of two vectors is smaller than or equal to the product of their magnitudes

$$
\left[f(\tau)-f\left(t_{k-1}\right)\right]^{\top} v(\tau) \leq\left|f(\tau)-f\left(t_{k-1}\right)\right||v(\tau)| .
$$

From (9) and (8)

$$
\left[f(\tau)-f\left(t_{k-1}\right)\right]^{\top} v(\tau) \leq \frac{\sigma_{0}(\mathbf{B})}{2 T}\left|x(\tau)-x\left(t_{k-1}\right)\right||v(\tau)| .
$$

From Conditions 2) and 3), we can prove that the distance between $x(\tau)$ and $x\left(t_{k-1}\right)$ is bounded (see Appendix)

$\left|x(\tau)-x\left(t_{k-1}\right)\right| \leq|v(\tau)|\left(\tau-t_{k-1}\right)+\frac{\sigma_{0}(\mathbf{S})}{2 T \sigma_{0}(\mathbf{B})}\left(\tau-t_{k-1}\right)^{2}$

Accounting for the computational delay, for $t_{k} \leq \tau<t_{k+1}$

$$
\left|x(\tau)-x\left(t_{k-1}\right)\right| \leq 2 T|v(\tau)|+\frac{2 T \sigma_{0}(\mathbf{S})}{\sigma_{0}(\mathbf{B})} .
$$


From (10) and from the above, we have

$$
\begin{aligned}
{[f(\tau)} & \left.-f\left(t_{k-1}\right)\right]^{\top} v(\tau) \\
& \leq \frac{\sigma_{0}(\mathbf{B})}{2 T}\left(2 T|v(\tau)|+\frac{2 T \sigma_{0}(\mathbf{S})}{\sigma_{0}(\mathbf{B})}\right)|v(\tau)| \\
& =\sigma_{0}(\mathbf{B})|v(\tau)|^{2}+\sigma_{0}(\mathbf{S})|v(\tau)| .
\end{aligned}
$$

Given that $\sigma_{0}(\mathbf{B})|v(\tau)|^{2} \leq v(\tau)^{\top} \mathbf{B} v(\tau)$ and that $\sigma_{0}(\mathbf{S})|v(\tau)|^{2} \leq v(\tau)^{\top} \mathbf{S} v(\tau),(13)$ yields

$$
\left[f(\tau)-f\left(t_{k-1}\right)\right]^{\top} v(\tau) \leq v(\tau)^{\top} \mathbf{B} v(\tau)+v(\tau)^{\top} \frac{\mathbf{S}}{|v(\tau)|} v(\tau) .
$$

$f\left(t_{k-1}\right)$ can be replaced by $f_{v}(\tau)$ in the above inequality

$$
\left[f(\tau)-f_{v}(\tau)\right]^{\top} v(\tau) \leq v(\tau)^{\top} \mathbf{B} v(\tau)+v(\tau)^{\top} \frac{\mathbf{S}}{|v(\tau)|} v(\tau) .
$$

Integrating both sides of (15) gives

$$
\begin{aligned}
\int_{0}^{t}[f(\tau)- & \left.f_{v}(\tau)\right]^{\top} v(\tau) d \tau \\
& \leq \int_{0}^{t} v(\tau)^{\top} \mathbf{B} v(\tau)+v(\tau)^{\top} \frac{\mathbf{S}}{|v(\tau)|} v(\tau) d \tau
\end{aligned}
$$

that is

$$
\int_{0}^{t}\left[f_{v}(\tau)-f(\tau)+\mathbf{B} v(\tau)+\mathbf{S} \frac{v(\tau)}{|v(\tau)|}\right]^{\top} v(\tau) d \tau \geq 0 .
$$

Condition (17) is the passivity condition for the subsystem of Fig. 4 and for $E(0)=0$, hence, under the conditions stated, the subsystem of Fig. 4 is passive for some $T>0$.

Theorem 1 implies that the passivity of the subsystem of Fig. 4, or equivalently, the passivity of the combination of a dissipative haptic device with a virtual environment representing a passive system $f(t)$ can be achieved by a sufficiently high update rate.

Conditions 1) and 2) in Theorem 1 are related to the minimum rate for passivity. Condition 2) is easily satisfied for feasible acceleration ranges (like not hitting the device). Condition 1) shows that the minimum rate depends on the virtual environment and on the dissipation given by the device. Take the example of a linear virtual environment $f(t)=\mathbf{A} x(t)$ where $\mathbf{A}$ is the stiffness matrix of a multidimensional virtual wall with point contact. The first condition is satisfied when

$$
\sigma_{n}(\mathbf{A}) \leq \frac{\sigma_{0}(\mathbf{B})}{2 T}
$$

The largest singular value $\sigma_{n}(\mathbf{A})$ can be viewed as the wall stiffness in the direction of maximum stiffness, and $\sigma_{0}(\mathbf{B})$ can be viewed as the device damping in the direction that provides the least of it. Notice that this result specializes to the formula found in [7] for the one-dimensional case. The two results differ by

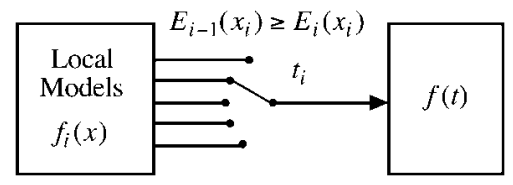

Fig. 5. Haptic rendering with local models.

a factor, because in [7], the computational delay was not accounted for, and the device and the user's dynamics were assumed to be linear.

It should be mentioned that a high sampling rate has no direct effect on the impact of measurement noise. Measurement and quantization noise, of course, can threaten the passivity of any digital system. The effect of a bounded measurement noise on the haptic interaction system of Fig. 3 can be analyzed using an approach similar to that used in the proof of Theorem 1, but is beyond the scope of this paper.

\section{PAssive Response From Local Models}

Recall that all systems and subsystems share the same flow variable $v(t)$ to denote the velocity of the contact interface between an operator and the device end-effector. For simplicity, from now on, we will name any block by the name of its associated effort variable.

We now show that $f(t)$, the continuous-time realization of a virtual environment, can be passively recreated in terms of local passive models, each activated for a certain time period, as in Fig. 5.

Theorem 2: Let

$$
f(t)=f_{i}(x), \quad t_{i} \leq t<t_{i+1} \quad \forall i>0
$$

where $i$ denotes a time slot from $t_{i}$ to $t_{i+1}$. If

1) $\nabla \times f_{i}(x)=0$, the $f_{i}(x)$ 's are static conservative fields

2) $E_{i}(x) \geq 0$, where $E_{i}(x)=\int_{x_{0}}^{x} f_{i}(y)^{\top} d y, \forall x \in$ $\mathbb{R}^{3}, x_{0}=x(0)$, that is, the $f_{i}(x)$ 's are passive

3) $E_{i}\left(x_{i}\right) \leq E_{i-1}\left(x_{i}\right), x_{i}=x\left(t_{i}\right)$, the potential energy of the virtual environment does not increase at the moment of activation

then system $f(t)$ is passive.

Proof: The passivity condition for system $f(t)$ is

$$
E(t)=\int_{0}^{t} f(\tau)^{\top} v(\tau) d \tau \geq 0
$$

For $t_{n} \leq t \leq t_{n+1} \forall n \geq 0, E(t)$, can be written in terms of $f_{i}$ 's

$$
\begin{aligned}
E(t) & =\sum_{i=0}^{n-1} \int_{t_{i}}^{t_{i+1}} f_{i}(x(\tau))^{\top} v(\tau) d \tau+\int_{t_{n}}^{t} f_{n}(x(\tau))^{\top} v(\tau) d \tau \\
E_{i} & \left(x_{i+1}\right)-E_{i}\left(x_{i}\right) \\
& =\int_{t_{i}}^{t_{i+1}} f_{i}(x(\tau))^{\top} v(\tau) d \tau, \quad \text { because } f_{i} \text { is static } \\
& =\int_{x_{i}}^{x_{i+1}} f_{i}(y)^{\top} d y, \quad \text { because } f_{i} \text { is conservative. }
\end{aligned}
$$




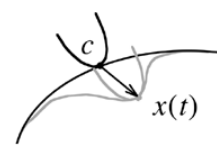

Fig. 6. A tool against an object forms a contact surface. The deflection $\delta$ at point $c$, a point of initial contact on the surface of an undeformed object (in black outline), is found from the difference between the new position $x(t)$ of $c$ on the tool once interaction occurs, and its initial value $c$. When the object deforms (in grey outline), a force response is produced that is characteristic of the body, of the tool, and of $c$.

Using (22), (21) can be written

$$
\begin{aligned}
E(t) & =\sum_{i=0}^{n-1}\left[E_{i}\left(x_{i+1}\right)-E_{i}\left(x_{i}\right)\right]+E_{n}(x)-E_{n}\left(x_{n}\right) \\
& =\sum_{i=1}^{n} E_{i-1}\left(x_{i}\right)-\sum_{i=0}^{n-1} E_{i}\left(x_{i}\right)-E_{n}\left(x_{n}\right)+E_{n}(x) \\
& =\sum_{i=1}^{n}\left[E_{i-1}\left(x_{i}\right)-E_{i}\left(x_{i}\right)\right]+E_{n}(x) .
\end{aligned}
$$

Then we get from above and from Conditions 2) and 3)

$$
E(t) \geq E_{n}(x) \geq 0
$$

therefore, system $f(t)$ is passive.

Theorem 2 provides an approach to create a passive environment from a succession of local passive models, where these models are constructed from a collection of locally defined static conservative force fields.

\section{Tool Contact With a Deformable Body}

In this section, we apply Theorems 1 and 2 to the high-fidelity haptic rendering of tool contact with a deformable body. Following the technique introduced in [19], the local models, corresponding to static conservative force fields, are constructed from actual force responses known at a discrete set of locations of an undeformed body [16]. These responses can be obtained with a variety of methods, for example, from actual measurement tests, from accurate offline numerical simulations, or from analytical solutions. The choice of a particular method is of no consequence here.

\section{A. Local Models}

We consider simplified local models designed to represent the behavior of inhomogeneous bodies while ignoring the dependency of the rotation of response coordinates on deflection. It could be included, if desired. The response of a given tool interacting at a finite number of points on the surface of the body is known. We call these points nodes. Sliding behavior is represented in terms of presliding displacements [12].

The haptic device measures $x(t)$, the position of the tip of a virtual tool. Point $c$ represents the position of the initial contact of the tool on the surface of the deformable body at rest. The deflection vector at point $c$ is $\delta=x-c$ (see Fig. 6).

The nodes distributed on the surface of the body define a triangulation that can be used to represent the geometry of the body at rest as a set of triangular elements. In the case of a single tool, (a)

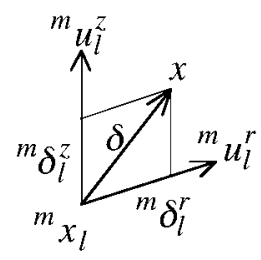

(b)

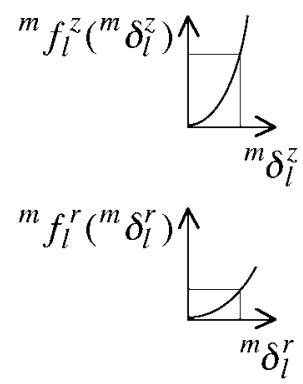

Fig. 7. (a) Deflection in local coordinates. (b) Response vector components.

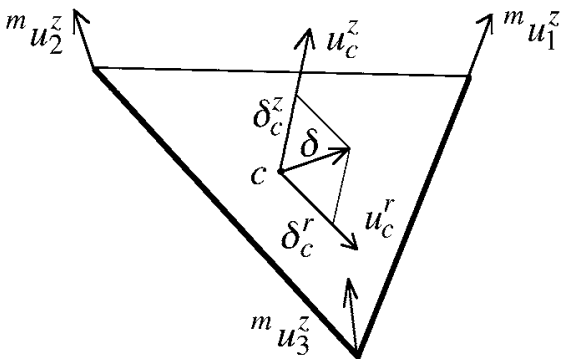

Fig. 8. Local coordinates at $c$. The normal vector $u_{c}^{z}$ points inside the body.

the geometry of the deformed body is irrelevant for haptics, only the force responses are relevant.

\section{B. Response of a Deformable Body}

The force response to contact at each node $l$ of element $m$ is represented in local coordinates. Each node is labeled ${ }^{m} x_{l}$. The unit vectors $m_{u_{l}^{z}}^{z}$ and $m_{u_{l}^{r}}^{r}$ with origin at $m_{x_{l}}$ are such that ${ }^{m} \delta_{l}={ }^{m} \delta_{l}^{z} m_{u_{l}}^{z}+{ }^{m} u_{l}^{r}{ }^{m} \delta_{l}^{r}$, as shown in Fig. 7(a). The quantities $m_{l} f_{l}^{z}(\cdot)$ and ${ }^{m} f_{l}^{r}(\cdot)$ are the components of the force responses, as in Fig. 7(b). The responses are derived from actual force responses (precalculated or premeasured) using appropriate approximations [19]. Given ${ }^{m} x_{l}$ and $x$, the force response at node $l$ of element $m$ is

$$
{ }^{m} f_{l}(\delta)={ }^{m} f_{l}^{r}\left({ }^{m} \delta_{l}^{r}\right){ }^{m} u_{l}^{r}+{ }^{m} f_{l}^{z}\left({ }^{m} \delta_{l}^{z}\right)^{m} u_{l}^{z} .
$$

\section{Interpolated Response}

When $c$ is inside an element $m$, coordinates are defined at point $c$ with a unit vector $u_{c}^{z}$ found by interpolation of unit vectors at each node. The unit vector $u_{c}^{r}$ is such that $\delta=\delta_{c}^{z} u_{c}^{z}+$ $\delta_{c}^{r} u_{c}^{r}$, as in Fig. 8. Call $m_{n_{l}}(\cdot)$ the interpolation function for node $l$ of element $m$

$$
u_{c}^{z}=\sum_{l=1,2,3} m_{n_{l}}(c)^{m_{u}} u_{l}^{z}
$$

the vector can then be normalized for more accuracy.

A common interpolation choice used in computational methods for continuous mechanics is based on natural coordinates. We adopt it here. Within one element of area ${ }^{m} A$, these coordinates are given by the areas ${ }^{m} A_{l}$ of the triangles defined by the contact point and the three vertices, as in Fig. 9(a)

$$
m_{n_{l}(c)}=\frac{m_{A_{l}}(c)}{m_{A}} .
$$


Note that the vector $u_{c}^{z}$ is normally different from the vector normal to the surface of the element.

A key property of the interpolation approach is to ensure continuity of coordinate change over the surface of the body. Refering to Fig. 9(b), the force-deflection response at $c$ is obtained from

$$
f_{c}(\delta)=f_{c}^{z}\left(\delta_{c}^{z}\right) u_{c}^{z}+f_{c}^{r}\left(\delta_{c}^{r}\right) u_{c}^{r}
$$

where $f_{c}^{z}(\cdot)$ and $f_{c}^{r}(\cdot)$ each are also interpolated from three precalculated force-deflection responses

$$
\begin{aligned}
& f_{c}^{z}(\cdot)=\sum_{l=1,2,3} m_{n_{l}}(c)^{m} f_{l}^{z}(\cdot) \\
& f_{c}^{r}(\cdot)=\sum_{l=1,2,3} m_{n_{l}(c)^{m} f_{l}(\cdot) .}
\end{aligned}
$$

During a time slot $i$, a local model $f_{i}(x)$ is defined, given a point of initial contact $c_{i}$

$$
f_{i}(x)=f_{c_{i}}(\delta)
$$

The $f_{i}$ 's are now defined for any initial contact points during a time slot.

\section{Conservation and Passivity of Local Models}

For each local model $f_{i}(x)$, we have the following. 1) Conservation

$$
\begin{aligned}
\nabla \times f_{i}(x) & =\nabla \times f_{c_{i}}(\delta) \\
& =\nabla \times\left[f_{c_{i}}^{r}\left(\delta_{c_{i}}^{r}\right) u_{c_{i}}^{r}+f_{c_{i}}^{z}\left(\delta_{c_{i}}^{z}\right) u_{c_{i}}^{z}\right] \\
& =0 .
\end{aligned}
$$

2) Passivity

$$
\begin{aligned}
E_{i}(x)= & \int_{0}^{x} f_{i}(y)^{\top} d y=\int_{0}^{\delta_{c_{i}}} f_{c_{i}}(\beta) d \beta \\
= & \int_{0}^{\delta_{c_{i}}^{r}} f_{c_{i}}^{r}\left(\beta^{r}\right) d \beta^{r}+\int_{0}^{\delta_{c_{i}}^{z}} f_{c_{i}}^{z}\left(\beta^{z}\right) d \beta^{z} \\
= & \int_{0}^{\delta_{c_{i}}^{r}}\left[\sum_{l=1,2,3} m_{n_{l}}\left(c_{i}\right)^{m} f_{l}\left(\beta^{r}\right)\right] d \beta^{r} \\
& +\int_{0}^{\delta_{c_{i}}^{z}}\left[\sum_{l=1,2,3} m_{n_{l}}\left(c_{i}\right)^{m} f_{l}\left(\beta^{z}\right)\right] d \beta^{z} \\
= & \sum_{l=1,2,3} m_{n_{l}\left(c_{i}\right)}\left[\int_{0}^{\delta_{c_{i}}^{r}}{ }^{m} f_{l}\left(\beta^{r}\right) d \beta^{r}\right. \\
& \left.+\int_{0}^{\delta_{c_{i}}^{z}}{ }^{m} f_{l}\left(\beta^{z}\right) d \beta^{z}\right] .
\end{aligned}
$$

Thus, if ${ }^{m} f_{l}\left(\beta^{z}\right) \beta^{z} \geq 0$ and ${ }^{m} f_{l}\left(\beta^{r}\right) \beta^{r} \geq 0 \forall \beta^{z}$ and $\forall \beta^{r}$, then $E_{i}(x) \geq 0$.
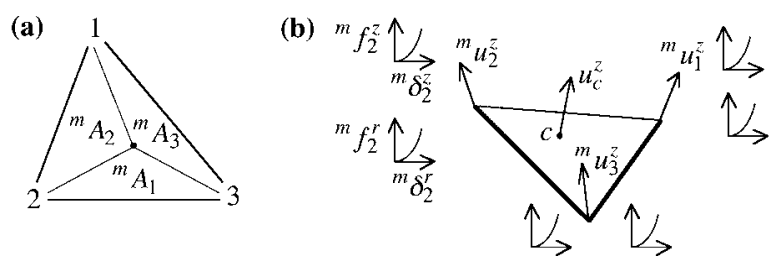

Fig. 9. (a) A point inside a triangle defines three areas labeled as indicated. (b) For each triangular element $m$, the vertices are associated to ${ }^{m} u_{l}^{z}, l=1,2,3$ and to two response components ${ }^{m} f_{l}^{z}$ and ${ }^{m} f_{l}^{r}$.

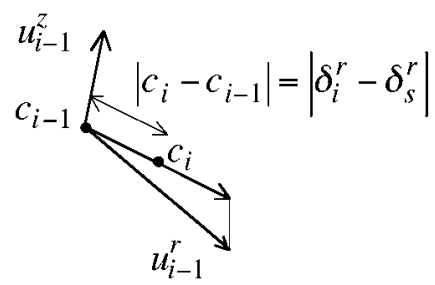

Fig. 10. Vector $u_{i-1}^{r}$ is projected onto the element surface to define the direction of the movement of $c$. If $\delta_{i-1}^{r}$ reaches $\delta_{s}^{r}, c$ slides by $\left|\delta_{i}^{r}-\delta_{s}^{r}\right|$.

\section{E. Passive Activation of Local Models}

A sequence of models is activated when $c$ moves on the surface of the body. Condition 3) of Theorem 2 should be satisfied to ensure a passive response. Activation of a new model occurs in one of two cases: at the instant of a collision and during sliding.

1) Collision: A collision occurs when the tool tip meets the body at rest. Because of the discrete nature of the simulation, the tool tip will almost never be located exactly on the nominal surface when a local model is activated at time $t_{i}$. To ensure passivity, the contact point $c_{i}$ is obtained by projecting $x_{i}$ over the surface of the body. From time $t_{i}$ and until the time of the next collision, $\delta$ is adjusted by replacing it by $\delta-\delta_{c_{i}}^{0}$, where $\delta_{c_{i}}^{0}$ is the value of $\delta_{c_{i}}$ before adjustment. This modification of the "relaxed state" ensures that $E_{i}\left(x_{i}\right)=E_{i}(0)=0$, which is a necessary condition for passivity required by Theorem 1 [17]. A collision at time $t_{i}$ is accepted only if both components of $\delta$ adjusted as a result of the previous collision are smaller than or equal to zero. This ensures that $E_{i-1}\left(x_{i}\right)=0$. Therefore, $E_{i}\left(x_{i}\right)=E_{i-1}\left(x_{i}\right)=0$ for any $t_{i}$ immediately after a collision.

2) Sliding: Let $\delta_{i-1}^{r}$ be $\delta^{r}$ at $i-1$, and let $\delta_{s}^{r}$ be the limiting sliding deflection such that $f_{i-1}^{r}\left(\delta_{s}^{r}\right)=\mu f_{i-1}^{z}\left(\delta_{i-1}^{z}\right)$ where $\mu$ is the limiting friction coefficient. ${ }^{1}$ In the presliding model [12], sliding occurs when $\delta_{i-1}^{r}>\delta_{s}^{r}$. When $c$ moves over the element surface, a new model $i$ is activated. The movement is in the direction of the projection of $u_{i-1}^{r}$ over the element surface, and its magnitude is $\left|\delta_{i}^{r}-\delta_{s}^{r}\right|$, see Fig. 10. The energy change $E_{i}\left(x_{i}\right)-E_{i-1}\left(x_{i-1}\right)$ results from three steps. First, shortening of $\delta_{i-1}^{r}$ to $\delta_{i}^{r}$; second, coordinates rotation; and third,

${ }^{1}$ This presliding distance is not to be confused with the presliding observed with hard materials in contact, which is measured in $\mu \mathrm{m}$ [3]. For soft materials, it can be large and depends mostly on the material properties and on its support. Consider, for example, the case of palpation. The skin can deform over large distances before slip occurs. Applying Amontons' law is equivalent to assuming that $\mu$ is invariant with the contact surface. 
change of force-deflection response. In the first step, the energy decreases due to sliding friction. The change of energy at the second and third steps depends on the force-deflection responses and the local coordinates at nodes. If the energy loss of the first step overcomes the possible energy gain of the second and third steps, then passive activation is obtained. It is also possible to manipulate force-deflection responses such that the overall energy change at the second and third steps become zero. At any rate, this level of sophistication may not be needed for most applications.

\section{MultiRate Simulation}

\section{A. Implementation}

Condition 1) of Theorem 1 is met if the sampling rate is sufficient to satisfy

$$
\left|f_{c}(\alpha)-f_{c}(\beta)\right|<\frac{\sigma_{0}(\mathbf{B})}{2 T}|\alpha-\beta| \quad \forall \alpha, \beta, c .
$$

In order to increase the rate of force update, it is possible to perform an element search in a separate process running at a low rate. The role of the low-rate process is then to supply the vertex locations, the $u_{z}$ of these vertices, and the force deflections of the active element, i.e., the element which carries the contact point at a given time. The active element is determined using an interference-detection method. Since the elements are large relative to a typical tool speed, this can be done at low rate; $100 \mathrm{~Hz}$ is sufficient (moving at $1 \mathrm{~m} / \mathrm{s}$ over $10-\mathrm{mm}$ patches).

Element delivery is accomplished with dual-threaded code communicating by a first-in first-out (FIFO) queue. Two cases can arise: if $c$ belongs to a neighboring patch found in the FIFO, a new element is accepted, otherwise, the rendering process carries on with same patch. An element replacement is allowed when the contact point $c$ reaches an edge of the active element. If a neighboring element is not available, $c$ remains at the edge till a new element is delivered by the low-rate process. This causes the tool to further deform the body instead of sliding over it. The sliding movement is later applied to a new element. Since the change in force at an element edge is continuous, even with updates at low rate, Condition 3) of Theorem 1 is satisfied.

\section{B. Illustrative Example}

The strategy of the last section was implemented to evaluate the passivity of the synthetized interactions. The test criterion was that the passivity margin of multirate rendering with local models should be close to the passivity margin of high-rate rendering with a single model. Therefore, two experiments were performed: one with a simple environment, and other with a complex environment.

The forces were generated by a PenCat/Pro haptic device (Immersion Canada, Inc.) for both environments. This device was powered by Lorentz flat-coil actuators driving the end-effector in a single stage via a planar linkage. These actuators were, in turn, powered by current amplifiers. As a result, the device had very little damping and friction, a best case for fidelity, but a

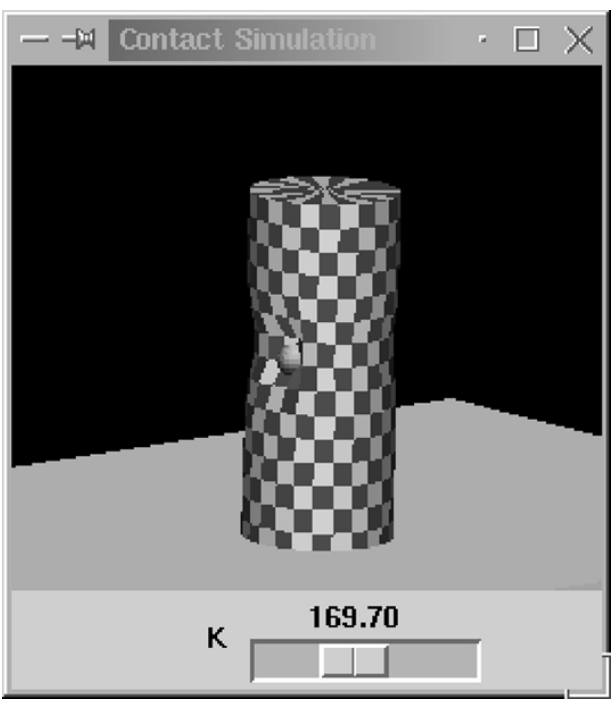

Fig. 11. Tool-contact simulation with a deformable cylindrical object. A cursor enabled adjusting the response interactively.

worse case for an inherent stability margin, in the sense discussed in Section III.

The simple environment was a nonlinear virtual flat wall

$$
f(x)= \begin{cases}0, & x \leq 0 \\ 100 k x^{2}, & 0<x \leq 0.005 \\ k(x-0.0025), & x>0.005\end{cases}
$$

The parameters were selected such that $k$ gave an appropriate passivity margin, as defined by Condition 1) of Theorem 1 .

In order to create repeatable experimental conditions, the handle of the device was loaded by an elastic rubber band stretched so as to create an equilibrium around $1 \mathrm{~N}$ inside the wall. Then, for a given $k$, a strong disturbance was applied by stretching the band $3 \mathrm{~cm}$ out of the wall and letting the device "sling shoot" into it without providing any damping. The smallest $k$ which created self-sustained oscillations defined a passivity margin. It was found that the interaction with the simple environment was passive for $k_{\max }=170 \mathrm{~N} / \mathrm{m}$ and for a rate of $2000 \mathrm{~Hz}$.

The multirate virtual environment constructed from local models was a tool interacting with a deformable cylindrical virtual body made of about 500 patches, as illustrated in Fig. 11. The local response at all nodes was

$$
f^{z}\left(\delta^{z}\right)= \begin{cases}0, & \delta^{z} \leq 0 \\ 100 k \delta^{2}, & 0<\delta^{z} \leq 0.005 \\ k\left(\delta^{z}-0.0025\right), & \delta^{z}>0.005\end{cases}
$$

The vectors $m_{u}^{z}$ at each mode were in the radial direction.

The simulation program consisted of two real-time threads running under RTLinux-3. One thread provided for rendering the forces and the other for finding the active element. The low-rate process was running at only $100 \mathrm{~Hz}$, yet the interaction was stable for $k_{\max }=170 \mathrm{~N} / \mathrm{m}$ with force-update rate at $2000 \mathrm{~Hz}$. This shows the same stability performance for both environments. Fig. 12 shows phase-plane plots of the trajectory just above and just below the passivity margin. 
(a)
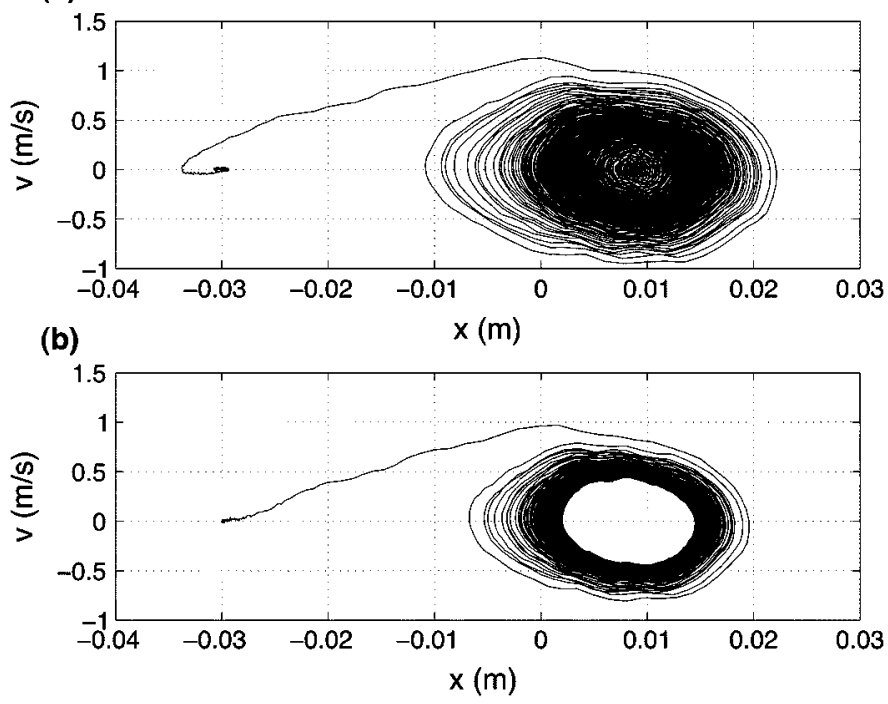

Fig. 12. (a) Locally stable response for $k=168 \mathrm{~N} / \mathrm{m}$. (b) Stable limit cycle when $k=179 \mathrm{~N} / \mathrm{m}$, indicating lack of passivity. The handle was released at $x=-0.03 \mathrm{~m}$ with zero velocity, and the wall begins at $x=0$. The rubber band was taut enough to cause the handle to hit it with $v=1 \mathrm{~m} / \mathrm{s}$.

\section{CONCLUSION}

The conditions for passivity of the force responses at the endeffector of a dissipative haptic device coupled a discrete-time realization of a passive virtual environment were obtained. It was found that a high update rate allows for a wider range of passive-force responses. A constructive approach for synthesizing the passive-force response of a nominally passive environment from locally defined force fields was described. This approach requires an energy-switching condition $E_{i}\left(x_{i}\right) \leq E_{i-1}\left(x_{i}\right)$ to be met over all local models. High-fidelity passive haptic rendering of tool contact in which passive, continuous force responses were generated from sparse actual tool responses was achieved. The corresponding haptic simulations did not have an active character despite the use of a direct-drive device having very little dissipation when the passivity conditions defined by Theorems 1 and 2 were met.

On-going work is concerned with the effect of quantization and measurement noise on the passivity of a haptic interaction. More general conditions for ensuring passive switching during sliding movement (Section V-E) are also investigated. Haptic rendering, in which forces depend on additional variables such as orientation of the haptic device and simulation states, are being developed [20].

\section{APPENDIX}

$$
\left|x(\tau)-x\left(t_{k-1}\right)\right|=\left|\int_{t_{k-1}}^{\tau} v(t) d t\right| \leq \int_{t_{k-1}}^{\tau}|v(t)| d t .
$$

If $v(t)$ is $C^{0}$ continuous, by the mean value theorem, there exists $\eta, t_{k-1}<\eta<\tau$, such that $v(t)=v(\tau)+a(\eta)(t-\tau)$. We can now write $|v(t)| \leq|v(\tau)|+|a(\eta)|(\tau-t)$. There also exists $a_{\max }$ such that $|a(\eta)|<a_{\max }, \forall \eta>0$, then

$$
|v(t)| \leq|v(\tau)|+a_{\max }(\tau-t) .
$$

From the above and from (36)

$$
\begin{aligned}
\left|x(\tau)-x\left(t_{k-1}\right)\right| & \leq \int_{t_{k-1}}^{\tau}\left[v(\tau) \mid+a_{\max }(\tau-t)\right] d t \\
& \leq|v(\tau)|\left(\tau-t_{k-1}\right)+\frac{1}{2} a_{\max }\left(\tau-t_{k-1}\right)^{2} .
\end{aligned}
$$

\section{ACKNOWLEDGMENT}

The authors would like to thank the reviewers for helpful comments.

\section{REFERENCES}

[1] R. J. Adams and B. Hannaford, "Stable haptic interaction with virtual environments," IEEE Trans. Robot. Autom., vol. 15, pp. 465-474, Jun. 1999.

[2] R. J. Anderson and M. Spong, "Bilateral control of teleoperators with time delay," IEEE Trans. Autom. Control, vol. 34, pp. 494-501, May 1989.

[3] B. Armstrong-Hélouvry, P. Dupont, and C. C. De Wit, "A survey of models, analysis tools, and compensation methods for the control of machines with friction," Automatica, vol. 30, no. 7, pp. 1083-1138, 1994.

[4] O. R. Astley and V. Hayward, "Multirate haptic simulation achieved by coupling finite element meshes through Norton equivalents," in Proc. IEEE Int. Conf. Robot. Autom., 1998, pp. 989-994.

[5] J. M. Brown and J. E. Colgate, "Passive implementation of multi-body simulations for haptic display," in Proc. Int. Mech. Eng. Congr. Exhibit., vol. 61, 1997, pp. 85-92.

[6] M. C. Cavusoglu and F. Tendick, "Multirate simulation for high-fidelity haptic interaction with deformable objects in virtual environments," in Proc. IEEE Int. Conf. Robot. Autom., 2000, pp. 2458-2464.

[7] J. E. Colgate and G. G. Schenkel, "Passivity of a class of sampled-data systems: Application to haptic interfaces," J. Robot. Syst., vol. 14, no. 1, pp. 37-47, 1997.

[8] S. Cotin, H. Delingette, and N. Ayache, "Real-time elastic deformations of soft tissues for surgery simulation," IEEE Trans. Vis. Comput. Graphics, vol. 5, pp. 62-73, Jan. 1999.

[9] R. E. Ellis, N. Sarkar, and M. A. Jenkins, "Numerical methods for the force reflection of contact," in ASME Trans. Dynam. Syst., Modeling, Control, vol. 119, 1997, pp. 768-774.

[10] B. Gillespie and M. Cutkosky, "Stable user-specific rendering of the virtual wall," in Proc. ASME Int. Mech. Eng. Conf. Expo., vol. DSC-58, 1996, pp. 397-406.

[11] B. Hannaford and J.-H. Ryu, "Stable haptic interaction with virtual environments," in IEEE Trans. Robot. Autom., vol. 18, Feb. 2002, pp. 1-10.

[12] V. Hayward and B. Armstrong, "A new computational model of friction applied to haptic rendering," in Experimental Robotics VI. ser. Lecture Notes in Control and Information Sciences, P. I. Corke and J. Trevelyan, Eds. New York: Springer-Verlag, 2000, vol. 250, pp. 403-412.

[13] V. Hayward and O. R. Astley, "Performance measures for haptic interfaces," in Robotics Research: The 7th International Symposium, G. Giralt and G. Hirzinger, Eds. New York: Springer-Verlag, 1996, pp. 195-207.

[14] D. L. James and D. K. Pai. (2001) A unified treatment of elastostatic and rigid contact simulation for real-time haptics. Haptics-e [Online], vol (1). Available: http://www.haptics-e.org/

[15] R. Lozano, B. Brogliato, O. Egeland, and B. Mashke, Dissipative Systems, Analysis and Control, Theory and Applications. New York: Springer-Verlag, 2000.

[16] M. Mahvash and V. Hayward, "High-fidelity haptic synthesis of contact with deformable bodies," IEEE Comput. Graph. Appl., vol. 24, pp. 48-55, Feb. 2004.

[17] — - "Passivity-based high-fidelity haptic rendering of contact," in Proc. IEEE Int. Conf. Robot. Autom., 2003, pp. 3722-3728.

[18] M. Mahvash, "Haptic rendering of tool contact and cutting," Ph.D. dissertation, McGill Univ., Montréal, QC, Canada, 2002.

[19] M. Mahvash, V. Hayward, and J. E. Lloyd, "Haptic rendering of tool contact," in Proc. Eurohaptics, 2002, pp. 110-115.

[20] M. Mahvash and V. Hayward. (2001) Haptic rendering of cutting, a fracture mechanics approach. Haptics-e [Online], vol (3). Available: http://www.haptics-e.org/

[21] B. Miller, J. E. Colgate, and R. A. Freeman, "Guaranteed stability of haptic systems with nonlinear virtual environments," IEEE Trans. Robot. Autom., vol. 16, pp. 712-719, Dec. 1999. 
[22] G. Niemeyer and J. J. Slotine, "Stable adaptive teleoperation," IEEE J. Ocean. Eng., vol. 16, pp. 152-162, Jan. 1991.

[23] S. E. Salcudean and N. R. Parker, "6-DOF desk-top voice-coil joystick," in Proc. 6th Annu. Symp. Haptic Interfaces Virtual Environ., Teleoper. Syst., vol. DSC-61, 1997, pp. 131-138.

[24] K. Salisbury, D. Brock, T. Massie, N. Swarup, and C. Zilles, "Haptic rendering: Programming touch interaction with virtual objects," in Proc. Symp. Interact. 3-D Graphics, 1995, pp. 123-130.

[25] S. Stramigioli, C. Secchi, A. J. van der Schaft, and C. Fantuzzi, "A novel theory for sample data system passivity," in Proc. IEEE/RSJ Int. Conf. Intell. Robots Syst., 2002, pp. 1936-1941.

[26] Y. Yokokohji, T. Imaida, and T. Yoshikawa, "Bilateral control with energy balance monitoring under time-varying communication delay," in Proc. IEEE Int. Conf. Robot. Autom., 2000, pp. 2684-2689.

[27] C. B. Zilles and J. K. Salisbury, "A constraint-based god object method for haptic display," in Proc. IEEE/RSJ Int. Conf. Intell. Robots Syst., vol. 3, 1995, pp. 146-151.

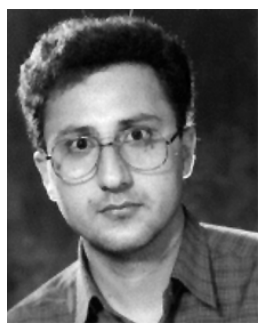

Mohsen Mahvash graduated from K. N. Toosi University of Technology, Tehran, Iran, and received a Master's degree in electrical engineering from Isfahan University of Technology, Isfahan, Iran. He received the Ph.D. degree in electrical and computer engineering from McGill University, Montréal, QC, Canada, in 2002.

$\mathrm{He}$ is Co-Founder and President of RealContact Inc., Montreal, QC, Canada. He is interested in surgical simulation, physics-based virtual reality, and control systems.

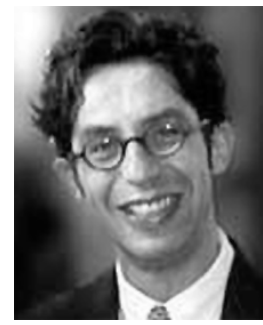

Vincent Hayward (M'84-SM'04) recieved the Diplôme d'Ingénieur in 1978 from the Ecole Centrale de Nantes, Nantes, France, and the Ph.D. degree in computer science in 1981 from the University of Paris, Paris, France.

He was Chargé de Recherches with CNRS, France, from 1983 to 1986, and is currently an Associate Professor of Electrical and Computer Engineering with McGill University, Montreal, QC, Canada. He is interested in haptic interfaces design and applications, perception, and robotics. He leads the Haptics Laboratory at McGill University, and is Director of the McGill Center for Intelligent Machines. His spin-off companies include Haptech in 1996, now Immersion Canada Inc., and RealContact, Montreal, QC, Canada, in 2002. He is the leader of a national project of IRIS, the Institute for Robotics and Intelligent Systems (Canada's Network of Centers of Excellence), and is a past member of the IRIS Research Management Board. He serves on the Governing Board of Haptics-e.

Dr. Hayward is a Co-Founder, Experimental Robotics Symposia and a past Associate Editor for the IEEE TRANSACTIONS ON ROBOTICS AND AUTOMATION. He served as Program Vice-Chair for the 1998 IEEE Conference on Robotics and Automation, and was Program Co-Chair for ISR2000. His awards include the NASA Space Act Tech Brief Award, recieved in 1991, and the E. (Ben) and Mary Hochhausen Award for Research in Adaptive Technology For Blind and Visually Impaired Persons, in 2002. 